\title{
Article \\ A Functional Characterization of Almost Greedy and Partially Greedy Bases in Banach Spaces
}

\author{
Pablo Manuel Berná ${ }^{*}+$ (D) and Diego Mondéjar ${ }^{\dagger}$ (D) \\ Departamento de Matemática Aplicada y Estadística, Facultad de Ciencias Económicas y Empresariales, \\ Universidad San Pablo-CEU, CEU Universities, 28003 Madrid, Spain; diego.mondejarruiz@ceu.es \\ * Correspondence: pablo.bernalarrosa@ceu.es \\ + These authors contributed equally to this work.
}

check for updates

Citation: Berná, P.M.; Mondéjar, D. A Functional Characterization of Almost Greedy and Partially Greedy Bases in Banach Spaces. Mathematics 2021, 9, 1827. https://doi.org/ $10.3390 /$ math 9151827

Academic Editor: Ioannis K. Argyros

Received: 30 June 2021

Accepted: 29 July 2021

Published: 2 August 2021

Publisher's Note: MDPI stays neutral with regard to jurisdictional claims in published maps and institutional affiliations.

Copyright: (c) 2021 by the authors. Licensee MDPI, Basel, Switzerland. This article is an open access article distributed under the terms and conditions of the Creative Commons Attribution (CC BY) license (https:// creativecommons.org/licenses/by/ $4.0 /)$.
Abstract: In 2003, S. J. Dilworth, N. J. Kalton, D. Kutzarova and V. N. Temlyakov introduced the notion of almost greedy (respectively partially greedy) bases. These bases were characterized in terms of quasi-greediness and democracy (respectively conservativeness). In this paper, we show a new functional characterization of these type of bases in general Banach spaces following the spirit of the characterization of greediness proved in 2017 by P. M. Berná and Ó. Blasco.

Keywords: greedy algorithm; nonlinear approximation; bases; Banach spaces

\section{Introduction and Background}

Assume that $(\mathbb{X},\|\cdot\|)$ is a Banach space over the field $\mathbb{F}=\mathbb{R}$ or $\mathbb{C}$. Throughout the paper, we assumed that $\mathcal{B}=\left(e_{n}\right)_{n=1}^{\infty}$ is a seminormalized Markushevich basis, that is there exists a unique sequence $\left(e_{n}^{*}\right)_{n=1}^{\infty} \subset \mathbb{X}^{*}$ such that:

- $\overline{\operatorname{span}\left(e_{n}: n \in \mathbb{N}\right)}=\mathbb{X}$;

- $e_{n}^{*}\left(e_{m}\right)=\delta_{n, m}$;

- $\quad$ if $e_{n}^{*}(f)=0$ for all $n \in \mathbb{N}$, then $f=0$;

- $\quad$ there are $c_{1}, c_{2}>0$ such that:

$$
0<c_{1}:=\inf _{n} \min \left\{\left\|e_{n}\right\|,\left\|e_{n}^{*}\right\|\right\} \leq \sup _{n} \max \left\{\left\|e_{n}\right\|,\left\|e_{n}^{*}\right\|\right\}=: c_{2}<\infty
$$

Hereinafter, by a basis for $\mathbb{X}$, we mean a seminormalized Markushevich basis. Under these conditions, for each $f \in \mathbb{X}$, we have that $f \sim \sum_{n=1}^{\infty} e_{n}^{*}(f) e_{n}$ where $\left(e_{n}^{*}(f)\right)_{n} \in c_{0}$. The support of $f \in \mathbb{X}$ is denoted by $\operatorname{supp}(f)$, where $\operatorname{supp}(f)=\left\{n \in \mathbb{N}:\left|e_{n}^{*}(f)\right| \neq 0\right\}$. Finally, we use the following notation: $\mathbb{X}_{f i n}$ is the subspace of $\mathbb{X}$ with the elements with finite support; if $f, g \in \mathbb{X}, f \cdot g=0$ means that $\operatorname{supp}(f) \cap \operatorname{supp}(g)=\varnothing, \tilde{f}=\left(e_{n}^{*}(f)\right)_{n \in \mathbb{N}}$ and $\|\tilde{f}\|_{\infty}=\sup _{n \in \mathbb{N}}\left|e_{n}^{*}(f)\right|$. Moreover, if $A$ and $B$ are finite sets of natural numbers, $A<B$ means that $\max _{n \in A} n<\min _{j \in B} j ; P_{A}$ is the projection operator, that is $P_{A}(f)=$ $\sum_{n \in A} e_{n}^{*}(f) e_{n}$; and $S_{k}$ is the partial sum of order $k$, that is $S_{k}(f)=P_{\{1, \cdots, k\}}(f)$.

In 1999, S. V. Konyagin and V. N. Temlyakov ([1])introduced one of the most studied algorithms in the field of nonlinear approximation, the so-called thresholding greedy algorithm: for $f \in \mathbb{X}$ and $m \in \mathbb{N}$, we define a greedy sum of order $m$ as:

$$
\mathcal{G}_{m}(f)[\mathbb{X}, \mathcal{B}]=\mathcal{G}_{m}(f):=\sum_{n \in A_{m}(f)} e_{n}^{*}(f) e_{n}
$$

where $A_{m}(f)$ is a greedy set of order $m$, that is $\left|A_{m}(f)\right|=m$ and:

$$
\min _{n \in A_{m}(f)}\left|e_{n}^{*}(f)\right| \geq \max _{n \notin A_{m}(f)}\left|e_{n}^{*}(f)\right| .
$$


The collection $\left(\mathcal{G}_{m}\right)_{m \in \mathbb{N}}$ is the thresholding greedy algorithm. As for every algorithm, one of the first questions that we can ask the audience is when the algorithm converges. To solve that question, S. V. Konyagin and V. N. Temlyakov introduced in [1] the notion of quasi-greediness.

Definition 1. We say that $\mathcal{B}$ is quasi-greedy if there is a positive constant $C$ such that:

$$
\left\|f-\mathcal{G}_{m}(f)\right\| \leq C\|f\|, \forall m \in \mathbb{N}, \forall f \in \mathbb{X} .
$$

The least constant verifying (1) is denoted by $C_{q}=C_{q}[\mathbb{X}, \mathcal{B}]$, and we say that $\mathcal{B}$ is $\mathrm{C}_{q}$-quasi-greedy.

Although this definition only talks about the boundedness of the greedy sums, P. Wojtaszczyk showed in [2] that quasi-greediness is equivalent to the convergence of the algorithm.

Theorem 1 ([2,3]). A basis $\mathcal{B}$ in a Banach (or quasi-Banach) space is quasi-greedy if and only if:

$$
\lim _{m \rightarrow+\infty}\left\|f-\mathcal{G}_{m}(f)\right\|=0, \forall f \in \mathbb{X}
$$

Then, quasi-greediness is the minimal condition in the convergence of the algorithm, but we are interested in others types of convergence. For instance, when does the algorithm produce the best possible approximation? To study this question, in [1], the authors introduced the notion of greediness: a basis $\mathcal{B}$ is greedy if there is a positive constant $C_{g}$ such that:

$$
\left\|f-\mathcal{G}_{m}(f)\right\| \leq C_{g} \inf \left\{\left\|f-\sum_{n \in B} a_{n} e_{n}\right\|: a_{n} \in \mathbb{F},|B| \leq m\right\}, \forall m \in \mathbb{N}, \forall f \in \mathbb{X} .
$$

There are several examples of greedy bases, for instance the canonical basis in the spaces $\ell_{p}$ with $1 \leq p<\infty$, the Haar system in $L_{p}((0,1))$ with $1<p<\infty$, or the trigonometric system in $L_{2}(\mathbb{T})$. To study greedy bases, S. V. Konyagin and V. N. Temlyakov gave a characterization in terms of unconditional and democratic bases, where a basis is unconditional if the projection operator is uniformly bounded, that is there is $K>0$ such that, for any finite set $A$,

$$
\left\|P_{A}(f)\right\| \leq K\|f\|, \forall f \in \mathbb{X} .
$$

Consider $A$ a finite set, and define the set of the collection of signs in $A, \mathcal{E}_{A}=\{\varepsilon=$ $\left.\left(\varepsilon_{n}\right)_{n \in A}:\left|\varepsilon_{n}\right|=1\right\}$, then take the indicator sum:

$$
1_{\varepsilon A}=1_{\varepsilon A}[\mathbb{X}, \mathcal{B}]:=\sum_{n \in A} \varepsilon_{n} e_{n}
$$

If $\varepsilon \equiv 1$, we use the notation $1_{A}$.

Definition 2 ([3-6]). We say that $\mathcal{B}$ is symmetric for the largest coefficients if there is a positive constant $C$ such that:

$$
\left\|f+1_{\varepsilon A}\right\| \leq C\left\|f+1_{\mathcal{\varepsilon}^{\prime} B}\right\|,
$$

for any pair of sets $|A| \leq|B|<\infty, A \cap B=\varnothing$, for any $f \in \mathbb{X}$ such that $\operatorname{supp}(f) \cap(A \cup B)=\varnothing$, $\left|e_{n}^{*}(f)\right| \leq 1$ for all $n \in \mathbb{N}$, and for any choice of signs $\varepsilon \in \mathcal{E}_{A}, \varepsilon^{\prime} \in \mathcal{E}_{B}$. The least constant verifying (2) is denoted by $\Delta=\Delta[\mathbb{X}, \mathcal{B}]$, and we say that $\mathcal{B}$ is $\Delta$-symmetric for the largest coefficients. If (2) is satisfied with the extra condition that $A<\operatorname{supp}(f) \cup B$, then we say that $\mathcal{B}$ is partially symmetric for the largest coefficients with constant $\Delta_{p c}$. 
Definition 3. We say that $\mathcal{B}$ is super-democratic if there is a positive constant $C$ such that:

$$
\left\|1_{\varepsilon A}\right\| \leq C\left\|1_{\eta B}\right\|
$$

for any pair of sets $A, B \subset \mathbb{N},|A| \leq|B|<\infty$ and for any choice of signs $\varepsilon \in \mathcal{E}_{A}, \eta \in \mathcal{E}_{B}$. The least constant verifying (3) is denoted by $\Delta_{S}=\Delta_{S}[\mathbb{X}, \mathcal{B}]$, and we say that $\mathcal{B}$ is $\Delta_{s}$-super-democratic.

If (3) is satisfied for $A<B$, we say that $\mathcal{B}$ is $\Delta_{s c}$-super-conservative.

If (3) is satisfied for $\varepsilon \equiv \eta \equiv 1$, we say that $\mathcal{B}$ is $\Delta_{d}$-democratic, and if in addition, $A<B$, we say that $\mathcal{B}$ is $\Delta_{c}$-conservative.

With these definitions, we can find the following characterizations of greedy bases.

Theorem 2. Assume that $\mathcal{B}$ is a basis in a Banach space $\mathbb{X}$ :

- $\mathcal{B}$ is greedy if and only if $\mathcal{B}$ is democratic and unconditional (see [1]). Moreover,

$$
\max \left\{K, \Delta_{d}\right\} \leq C_{g} \leq K+K^{2} \Delta_{d} ;
$$

- $\quad \mathcal{B}$ is greedy if and only if $\mathcal{B}$ is super-democratic and unconditional (see [7]). Moreover,

$$
\max \left\{K, \Delta_{s}\right\} \leq C_{g} \leq K+K \Delta_{s} ;
$$

- $\quad \mathcal{B}$ is greedy if and only if $\mathcal{B}$ is symmetric for the largest coefficients and unconditional (see [6]). Moreover,

$$
\max \left\{K, \Delta_{d}\right\} \leq C_{g} \leq K \Delta .
$$

The last two characterizations were studied with the objective to improve the boundedness constant of greedy bases. Moreover, all the characterizations were given under the assumption of unconditionality and one of the democracy-like properties, but in [8], we found a new and interesting property that is very useful to give a new characterization of greediness (see [8] Corollary 1.8). This property is the so-called Property (Q): there is a $C>0$ such that:

$$
\left\|f+1_{A}\right\| \leq C\left\|f+g+1_{B}\right\|
$$

for any $|A|=|B|<\infty, A \cap B=\varnothing$ and $f, g \in \mathbb{X}_{\text {fin }}$ such that $\operatorname{supp}(f) \cap \operatorname{supp}(g)=\varnothing$, $\|\tilde{f}\|_{\infty} \leq 1$ and $\operatorname{supp}(f+g) \cap(A \cup B)=\varnothing$.

In that paper, we focused our attention in a closed inequality to characterize the so-called almost greedy and partially greedy bases.

Definition 4 ([4]). We say that $\mathcal{B}$ is almost greedy if there is a positive constant $C$ such that:

$$
\left\|f-\mathcal{G}_{m}(f)\right\| \leq C \inf \left\{\left\|f-P_{B}(f)\right\|:|B| \leq m\right\}, \forall m \in \mathbb{N}, \forall f \in \mathbb{X} .
$$

The least constant verifying (4) is denoted by $C_{a l}=C_{a l}[\mathbb{X}, \mathcal{B}]$, and we say that $\mathcal{B}$ is $C_{a l}$-almost greedy.

Definition $\mathbf{5}([4,5])$. We say that $\mathcal{B}$ is partially greedy if there is positive constant $C$ such that:

$$
\left\|f-\mathcal{G}_{m}(f)\right\| \leq C \inf _{k \leq m}\left\|f-S_{k}(f)\right\|, \forall m \in \mathbb{N}, \forall f \in \mathbb{X} .
$$

The least constant verifying (5) is denoted by $C_{p}=C_{p}[\mathbb{X}, \mathcal{B}]$, and we say that $\mathcal{B}$ is $C_{p^{-}}$ partially greedy.

Remark 1. In [4], the condition of partial greediness was introduced as follows:

$$
\left\|f-\mathcal{G}_{m}(f)\right\| \leq C\left\|f-S_{m}(f)\right\|, \forall m \in \mathbb{N}, \forall f \in \mathbb{X} .
$$


Under the condition of Schauder bases, (6) and (5) are equivalent notions, and in [5], the authors proved that if (6) is satisfied with $C=1$, then the basis is partially greedy.

Of course, every greedy basis is almost greedy and every almost greedy basis is partially greedy. One example of an almost greedy basis that is not greedy is the Lindenstrauss basis in $\ell_{1}$ ([9]). Recently, one basis that is partially greedy and not almost greedy was presented in ([10] Proposition 6.10).

It is well known that a basis is almost greedy if and only if the basis is quasi-greedy and democratic and a basis is partially greedy if the basis is quasi-greedy and conservative $([4,10])$. Moreover, as for greedy bases, we have the following characterizations.

Theorem 3. Assume that $\mathcal{B}$ is a basis in a Banach space:

- $\mathcal{B}$ is almost greedy if and only if $\mathcal{B}$ is democratic and quasi-greedy ([4]). Moreover,

$$
\max \left\{C_{q}, \Delta_{d}\right\} \leq C_{a l} \leq 8 C_{q}^{4} \Delta_{d}+C_{q}+1 ;
$$

- $\mathcal{B}$ is almost greedy if and only if $\mathcal{B}$ is super-democratic and quasi-greedy ([7]). Moreover,

$$
\max \left\{C_{q}, \Delta_{s}\right\} \leq C_{a l} \leq C_{q}+C_{q} \Delta_{s} ;
$$

- $\quad \mathcal{B}$ is almost greedy if and only if $\mathcal{B}$ is symmetric for the largest coefficients and quasi-greedy ([7]). Moreover,

$$
\max \left\{C_{q}, \Delta\right\} \leq C_{a l} \leq C_{q} \Delta .
$$

Theorem $4([4,5])$. Assume that $\mathcal{B}$ is a basis in a Banach space:

- $\mathcal{B}$ is partially greedy if and only if $\mathcal{B}$ is conservative and quasi-greedy. Moreover,

$$
\max \left\{C_{q}, \Delta_{c}\right\} \leq C_{p} \leq C_{q}+C_{q}^{2}\left(1+C_{q}\right) \Delta_{c} ;
$$

- $\mathcal{B}$ is partially greedy if and only if $\mathcal{B}$ is super-conservative and quasi-greedy. Moreover,

$$
\max \left\{C_{q}, \Delta_{s c}\right\} \leq C_{p} \leq C_{q}+C_{q}\left(1+C_{q}\right) \Delta_{s c} ;
$$

- $\mathcal{B}$ is partially greedy if and only if $\mathcal{B}$ is partially symmetric for the largest coefficients and quasi-greedy. Moreover,

$$
\max \left\{C_{q}, \Delta_{p c}\right\} \leq C_{p} \leq C_{q} \Delta_{p c} .
$$

The purpose of this paper is to obtain a new characterization of almost greedy and partially greedy bases following the ideas of ([8] Corollary 1.8) for greedy bases.

Definition 6. We say that $\mathcal{B}$ has Property $(F)$ if there is a positive constant $C$ such that:

$$
\left\|f+1_{A}\right\| \leq C\left\|f+g+1_{B}\right\|,
$$

for any $A, B, f, g$ satisfying the following conditions:

(i) $|A| \leq|B|<\infty$ and $A \cap B=\varnothing$;

(ii) $f, g \in \mathbb{X}_{\text {fin }}, f \cdot g=0, \operatorname{supp}(f+g) \cap(A \cup B)=\varnothing,\|\tilde{f}\|_{\infty} \leq 1$, and $\|\tilde{f}\|_{\infty}$ $\leq \inf _{n \in \operatorname{supp}(g)}\left|e_{n}^{*}(g)\right|$.

The least constant verifying $(7)$ is denoted by $\mathcal{F}=\mathcal{F}[\mathbb{X}, \mathcal{B}]$, and we say that $\mathcal{B}$ has Property (F) with constant $\mathcal{F}$.

Furthermore, if (7) is satisfied with the extra condition that $A<\operatorname{supp}(g) \cup B$, we say that $\mathcal{B}$ has Property $\left(F_{p}\right)$ with constant $\mathcal{F}_{p}$. 
Definition 7. We say that $\mathcal{B}$ has Property $\left(F^{*}\right)$ if there is a positive constant $C$ such that:

$$
\|f+z\| \leq C\|f+y\|
$$

for any $f, z, y \in \mathbb{X}_{\text {fin }}$ satisfying the following conditions:

(i) $f \cdot z=0, f \cdot y=0, z \cdot y=0$;

(ii) $\max \left\{\|\tilde{f}\|_{\infty},\|\tilde{z}\|_{\infty}\right\} \leq 1$;

(iii) $|D| \geq|\operatorname{supp}(z)|$, where $D=\left\{n \in \operatorname{supp}(y):\left|e_{n}^{*}(y)\right|=1\right\}$;

(iv) $\inf _{n \in \operatorname{supp}(y)}\left|e_{n}^{*}(y)\right| \geq\|\tilde{f}\|_{\infty}$.

The least constant verifying (8) is denoted by $\mathcal{F}^{*}=\mathcal{F}^{*}[\mathbb{X}, \mathcal{B}]$, and we say that $\mathcal{B}$ has Property $\left(F^{*}\right)$ with constant $\mathcal{F}^{*}$.

Furthermore, if (8) is satisfied with the extra condition that $\operatorname{supp}(z)<\operatorname{supp}(f+y)$, we say that $\mathcal{B}$ has Property $\left(F_{p}^{*}\right)$ with constant $\mathcal{F}_{p}^{*}$.

The main theorems that we prove are the following.

Theorem 5. Let $\mathcal{B}$ be a basis in a Banach space $\mathbb{X}$ :

(a) If $\mathcal{B}$ is almost greedy with constant $C_{a l}$, then $\mathcal{B}$ has Property $\left(F^{*}\right)$ with constant $\mathcal{F}^{*} \leq$ $C_{a l}\left(1+2 C_{a l}\right)$;

(b) If $\mathcal{B}$ has Property $\left(F^{*}\right)$ with constant $\mathcal{F}^{*}$, then the basis is almost greedy with constant $C_{a l} \leq\left(\mathcal{F}^{*}\right)^{2}$

Theorem 6. Let $\mathcal{B}$ be a basis in a Banach space $\mathbb{X}$ :

(a) If $\mathcal{B}$ is partially greedy with constant $C_{p}$, then $\mathcal{B}$ has Property $\left(F_{p}^{*}\right)$ with constant $\mathcal{F}_{p}^{*} \leq$ $C_{p}\left(1+2 C_{p}\right)$;

(b) If $\mathcal{B}$ has Property $\left(F_{p}^{*}\right)$ with constant $\mathcal{F}_{p}^{*}$, then the basis is partially greedy with constant $C_{p} \leq\left(\mathcal{F}_{p}^{*}\right)^{2}$

The structure of the paper is the following: In Section 2, we show some basics about Properties $(\mathrm{F})$ and $\left(\mathrm{F}^{*}\right)$. In Section 3, we prove Theorem 5. In Section 4 we give a brief summary about Properties $\left(\mathrm{F}_{p}\right)$ and $\left(\mathrm{F}_{p}^{*}\right)$. In Section 5 we prove Theorem 6, and finally, in Section 6, we give some density results that we use in the paper.

\section{Properties $(\mathrm{F})$ and $\left(\mathrm{F}^{*}\right)$}

This section is focused on the study of Properties $(F)$ and $\left(F^{*}\right)$. In fact, we show that these properties are equivalent. We show that we need some auxiliary lemmas about convexity.

Lemma 1 ([3] Corollary 2.3). Let $\mathbb{X}$ be a Banach space, and let $\mathcal{B}$ be a basis for $\mathbb{X}$ and J a finite set:

(i) For any scalars $\left(a_{j}\right)_{j \in J}$ with $0 \leq a_{j} \leq 1$ and any $g \in \mathbb{X}$,

$$
\left\|g+\sum_{j \in J} a_{j} e_{j}\right\| \leq \sup \left\{\left\|g+1_{A}\right\|: A \subseteq J\right\}
$$

(ii) For any scalars $\left(a_{j}\right)_{j \in J}$ with $\left|a_{j}\right| \leq 1$ and any $g \in \mathbb{X}$,

$$
\left\|g+\sum_{j \in J} a_{j} e_{j}\right\| \leq \sup _{\varepsilon \in \mathcal{E}_{J}}\left\|g+1_{\varepsilon J}\right\| .
$$

Lemma 2. Let $\mathcal{B}$ be a basis of a Banach space $\mathbb{X}$. Then,

$$
\sup _{\varepsilon \in \mathcal{E}_{A}}\left\|f+1_{\varepsilon A}\right\| \leq 5 \sup _{B \subseteq A}\left\|f+1_{B}\right\| .
$$


Proof. If $\mathbb{F}=\mathbb{R}$, following the result in ([8] Lemma 2.3), we know that:

$$
\sup _{\varepsilon_{n}= \pm 1}\left\|f+1_{\varepsilon A}\right\| \leq 3 \sup _{B \subseteq A}\left\|f+1_{B}\right\| .
$$

We prove now the result for the complex case. In that case,

$$
\begin{aligned}
1_{\varepsilon A} & =\sum_{n \in A} \operatorname{Re}\left(\varepsilon_{n}\right) e_{n}+i \sum_{n \in A} \operatorname{Im}\left(\varepsilon_{n}\right) e_{n} \\
& =\sum_{n \in A_{1}} \operatorname{Re}^{+}\left(\varepsilon_{n}\right) e_{n}-\sum_{n \in A_{2}} \operatorname{Re}^{-}\left(\varepsilon_{n}\right) e_{n} \\
& +i\left(\sum_{n \in A_{3}} \operatorname{Im}^{+}\left(\varepsilon_{n}\right) e_{n}-\sum_{n \in A_{4}} \operatorname{Im}^{-}\left(\varepsilon_{n}\right) e_{n}\right),
\end{aligned}
$$

where $A_{i}$ are the corresponding subsets of $A$. Then,

$$
\begin{aligned}
\left\|f+1_{\varepsilon A}\right\| & \leq\left\|f+\sum_{n \in A} \operatorname{Re}\left(\varepsilon_{n}\right) e_{n}\right\|+\left\|\sum_{n \in A} \operatorname{Im}\left(\varepsilon_{n}\right) e_{n}\right\| \\
& \leq\|f\|+\left\|f+\sum_{n \in A_{1}} \operatorname{Re}^{+}\left(\varepsilon_{n}\right) e_{n}\right\|+\left\|f+\sum_{n \in A_{2}} \operatorname{Re}^{-}\left(\varepsilon_{n}\right) e_{n}\right\| \\
& +\left\|f+\sum_{n \in A_{3}} \operatorname{Im}^{+}\left(\varepsilon_{n}\right) e_{n}\right\|+\left\|f+\sum_{n \in A_{4}} \operatorname{Im}^{-}\left(\varepsilon_{n}\right) e_{n}\right\| \\
& \leq 5 \sup _{B \subseteq A}\left\|f+1_{B}\right\| .
\end{aligned}
$$

Theorem 7. Let $\mathcal{B}$ be a basis in a Banach space $\mathbb{X}$. The basis is democratic (or symmetric for the largest coefficients) and quasi-greedy if and only if the basis has Property (F). Concretely:

(1) If $\mathcal{B}$ has Property $(F)$ with constant $\mathcal{F}$, then the basis is $C_{q}$-quasi-greedy and $\Delta_{d}$-democratic with:

$$
\max \left\{C_{q}, \Delta_{d}\right\} \leq \mathcal{F} ;
$$

(2) If $\mathcal{B}$ has Property $(F)$ with constant $\mathcal{F}$, then the basis is $C_{q}$-quasi-greedy and $\Delta$-symmetric for the largest coefficients with:

$$
C_{q} \leq \mathcal{F}, \Delta \leq 5\left(\mathcal{F}+4 \mathcal{F}^{2}+4 \mathcal{F}^{3}\right) ;
$$

(3) If $\mathcal{B}$ is $\Delta_{d}$-democratic and $C_{q}$-quasi-greedy, then the basis has Property $(F)$ with constant:

$$
\mathcal{F} \leq C_{q}\left(1+\left(1+C_{q}\right) \Delta_{d}\right) ;
$$

(4) If $\mathcal{B}$ is $\Delta$-symmetric for the largest coefficients and $C_{q}$-quasi-greedy, then the basis has Property (F) with constant:

$$
\mathcal{F} \leq 3 \Delta C_{q}
$$

Proof. First of all, we show (1). Assume that the basis has Property (F) with constant $\mathcal{F}$. To show that $\mathcal{B}$ is quasi-greedy, we take $f \in \mathbb{X}_{\text {fin }}$ with $t=\|\tilde{f}\|_{\infty}$ and $m \in \mathbb{N}$. Then, if we take in the definition of Property (F) $f^{\prime}=\frac{f}{t}-\mathcal{G}_{m}\left(\frac{f}{t}\right), g^{\prime}=\mathcal{G}_{m}\left(\frac{f}{t}\right)$, and $A=B=\varnothing$, since $\left\|\tilde{f}^{\prime}\right\|_{\infty} \leq \inf _{n \in \operatorname{supp}\left(g^{\prime}\right)}\left|e_{n}^{*}\left(g^{\prime}\right)\right|$, we obtain that:

$$
\left\|f-\mathcal{G}_{m}(f)\right\|=t\left\|f^{\prime}\right\| \leq \mathcal{F} t\left\|f^{\prime}+g^{\prime}\right\|=\mathcal{F}\|f\|,
$$

so the basis is quasi-greedy with $C_{q} \leq \mathcal{F}$ for elements with finite support. To obtain that $\mathcal{B}$ is quasi-greedy for any $f \in \mathbb{X}$, we use Corollary 1 . 
We prove now that the basis is democratic. For that, we take $C$ and $D$, two finite sets, such that $|C| \leq|D|$. Now, we perform the following decomposition:

$$
D=(D \cap C) \cup D_{1} \cup D_{2},
$$

where $\left|D_{1}\right|=|C \backslash D|$ and $D_{1} \cap D_{2}=\varnothing$. Hence, taking $f=1_{D \cap C}, g=1_{D_{2}}, A=C \backslash D$, and $B=D_{1}$,

$$
\left\|1_{C}\right\|=\left\|1_{C \cap D}+1_{C \backslash D}\right\| \leq \mathcal{F}\left\|1_{D \cap C}+1_{D_{2}}+1_{D_{1}}\right\|=\mathcal{F}\left\|1_{D}\right\| .
$$

Thus, $\mathcal{B}$ is democratic with $\Delta_{d} \leq \mathcal{F}$.

We now prove (2). We only have to show that $\mathcal{B}$ is symmetric for the largest coefficients. For that, take $f \in \mathbb{X}_{\text {fin }},\|\tilde{f}\|_{\infty} \leq 1, A \cap B=\varnothing,|A| \leq|B|<\infty$, $\operatorname{supp}(f) \cap(A \cup B)=\varnothing$, $\varepsilon \in \mathcal{E}_{A}$, and $\eta \in \mathcal{E}_{B}$. Using Lemmas 1 and 2, we only have to show that there is some absolute constant $C$ such that:

$$
\left\|f+1_{A^{\prime}}\right\| \leq C\left\|f+1_{\eta B}\right\|, \forall A^{\prime} \subseteq A .
$$

Of course, since Property (F) implies quasi-greediness with constant $C_{q} \leq \mathcal{F}$ by (1), if we take the element $h:=f+1_{\eta B}$ with $\|\tilde{f}\|_{\infty} \leq 1$, we have:

$$
\|f\|=\left\|h-\mathcal{G}_{|B|}(h)\right\| \leq \mathcal{F}\|h\|=\mathcal{F}\left\|f+1_{\eta B}\right\| .
$$

Furthermore, with respect to the set $A^{\prime}$, we can have the following:

$$
\left\|1_{A^{\prime}}\right\| \leq \mathcal{F}\left\|1_{B}\right\| \leq 4 \mathcal{F}^{2}\left\|1_{\eta B}\right\|
$$

where in the last inequality, we used ([11] Proposition 2.1.11) or ([3] Lemma 3.2). (These results affirm that for quasi-greedy bases, $\left\|1_{\varepsilon A}\right\| \leq 2 \kappa C_{q}\left\|1_{\eta A}\right\|$, for any $\eta, \varepsilon \in \mathcal{E}_{A}$ and any finite set $A$ with $\kappa=1$ if $\mathbb{F}=\mathbb{R}$ and $\kappa=2$ if $\mathbb{F}=\mathbb{C}$.)

Thus,

$$
\begin{aligned}
\left\|f+1_{A^{\prime}}\right\| & \leq\|f\|+\left\|1_{A^{\prime}}\right\| \underset{(10)+(11)}{\leq} \mathcal{F}\left\|f+1_{\eta B}\right\|+4 \mathcal{F}^{2}\left\|1_{\eta B}\right\| \\
& \leq\left(\mathcal{F}+4 \mathcal{F}^{2}\right)\left\|f+1_{\eta B}\right\|+4 \mathcal{F}^{2}\|f\| \\
& \leq\left(\mathcal{F}+4 \mathcal{F}^{2}+4 \mathcal{F}^{3}\right)\left\|f+1_{\eta B}\right\| .
\end{aligned}
$$

Finally, applying convexity,

$$
\left\|f+1_{\varepsilon A}\right\| \underset{\text { Lemma } 2}{\leq} 5 \sup _{A^{\prime} \subseteq A}\left\|f+1_{A^{\prime}}\right\| \leq 5\left(\mathcal{F}+4 \mathcal{F}^{2}+4 \mathcal{F}^{3}\right)\left\|f+1_{\eta B}\right\| .
$$

Therefore, the basis is symmetry for the largest coefficients for elements with finite support with constant:

$$
\Delta \leq 5\left(\mathcal{F}+4 \mathcal{F}^{2}+4 \mathcal{F}^{3}\right)
$$

Applying Lemma 5, the result follows for any $f \in \mathbb{X}$.

(3)Assume now that $\mathcal{B}$ is $C_{q}$-quasi-greedy and $\Delta_{d}$-democratic, and take $f, g \in \mathbb{X}_{\text {fin }}$ with $f \cdot g=0, \inf _{n \in \operatorname{supp}(g)}\left|e_{n}^{*}(g)\right| \geq\|\tilde{f}\|_{\infty}, A \cap B=\varnothing,|A| \leq|B|<\infty$, and $\operatorname{supp}(f+g) \cap$ $(A \cup B)=\varnothing$.

$$
\left\|f+1_{A}\right\| \leq\|f\|+\left\|1_{A}\right\| \leq\|f\|+\Delta_{d}\left\|1_{B}\right\|
$$

If we take $h:=f+g+1_{B}$, it is clear that $\operatorname{supp}\left(g+1_{B}\right)$ is a greedy set of $h$. Then, if $\mid \operatorname{supp}(g+$ $\left.1_{B}\right) \mid=n$

$$
\|f\|=\left\|h-\mathcal{G}_{n}(h)\right\| \leq C_{q}\|h\|=C_{q}\left\|f+g+1_{B}\right\|
$$


Since $\inf _{n \inf _{n \in \operatorname{supp}(g)}}\left|e_{n}^{*}(g)\right| \geq\|\tilde{f}\|_{\infty}$ and $\|\tilde{f}\|_{\infty} \leq 1$, we can decompose $g$ as $g=g_{1}+g_{2}$, where $\operatorname{supp}\left(g_{1}\right)=\left\{n \in \operatorname{supp}(g):\left|e_{n}^{*}(g)\right| \geq 1\right\}$ and $\operatorname{supp}\left(g_{2}\right)=\left\{n \in \operatorname{supp}(g):\left|e_{n}^{*}(g)\right|<\right.$ $1\}$. Then, if we take $u:=f+g_{2}+1_{B}, B$ is a greedy set for $u$ of order $k:=|B|$, and taking $v=u+g_{1}, \operatorname{supp}\left(g_{1}\right)$ is a greedy set of $v$ of order $p:=\left|\operatorname{supp}\left(g_{1}\right)\right|$. Thus,

$$
\begin{aligned}
\left\|1_{B}\right\| & =\left\|\mathcal{G}_{k}(u)\right\| \leq\left(1+C_{q}\right)\|u\|=\left(1+C_{q}\right)\left\|f+g_{2}+1_{B}\right\| \\
& =\left(1+C_{q}\right)\left\|v-\mathcal{G}_{p}(v)\right\| \leq\left(1+C_{q}\right) C_{q}\left\|f+g+1_{B}\right\| .
\end{aligned}
$$

Adding up (13) and (14) in (12), we obtain the result, that is the basis has Property (F) with $\mathcal{F} \leq C_{q}\left(1+\left(1+C_{q}\right) \Delta_{d}\right)$.

(4)Finally, assume that $\mathcal{B}$ is $\Delta$-symmetric for the largest coefficients and $C_{q}$-quasigreedy. Take $f, g, A$, and $B$ as in Property (F). Then,

$$
\begin{aligned}
\left\|f+1_{A}\right\| & \leq \Delta\left\|f+1_{B}\right\| \leq \Delta\left(\left\|f+g_{1}+1_{B}\right\|+\left\|g_{1}+f\right\|+\|f\|\right) \\
& \leq 3 C_{q} \Delta\left\|f+g+1_{B}\right\| .
\end{aligned}
$$

Thus, the basis has Property (F) with constant $\mathcal{F} \leq 3 C_{q} \Delta$.

Theorem 8. Let $\mathcal{B}$ be a basis in a Banach space $\mathbb{X}$. The basis has Property $(F)$ if and only if the basis has Property $\left(F^{*}\right)$. Moreover, if $\mathcal{F}$ and $\mathcal{F}^{*}$ are the constants of the corresponding properties, then:

$$
\mathcal{F} \leq \mathcal{F}^{*} \leq 5 \mathcal{F}\left(1+2 \mathcal{F}+8 \mathcal{F}^{2}\right) .
$$

Proof. Assume that we have Property $\left(\mathrm{F}^{*}\right)$ with constant $\mathcal{F}^{*}$, and take $f, g, A$, and $B$ as in Property (F), that is $f \cdot g=0, A \cap B=\varnothing,|A| \leq|B|, \operatorname{supp}(f+g) \cap(A \cup B)=\varnothing,\|\tilde{f}\|_{\infty} \leq 1$, and $\|\tilde{f}\|_{\infty} \leq \inf _{n \in \operatorname{supp}(g)}\left|e_{n}^{*}(g)\right|$. Taking $z=1_{A}$ and $y=g+1_{B}$ in Property $\left(\mathrm{F}^{*}\right), f, z$, and $y$ verify the conditions established in Property $\left(\mathrm{F}^{*}\right)$. Then,

$$
\left\|f+1_{A}\right\|=\|f+z\| \leq \mathcal{F}^{*}\|f+y\|=\mathcal{F}^{*}\left\|f+g+1_{B}\right\|,
$$

so the basis has Property (F) with $\mathcal{F} \leq \mathcal{F}^{*}$.

Assume now that we have Property (F), and take $f, y$, and $z$ in $\mathbb{X}_{\text {fin }}$ as in Property $\left(\mathrm{F}^{*}\right)$, that is $f \cdot z=0, f \cdot y=0, z \cdot y=0, \max \left\{\|\tilde{f}\|_{\infty},\|\tilde{z}\|_{\infty}\right\} \leq 1$, and $|\operatorname{supp}(z)| \leq|D|$ where $D=\left\{n:\left|e_{n}^{*}(y)\right|=1\right\} \mid$. Using Lemmas 1 and 2 , it is enough to prove that there exists $C_{1}>0$ such that:

$$
\left\|f+1_{A^{\prime}}\right\| \leq C_{1}\|f+y\|, \forall A^{\prime} \subseteq A,
$$

where $A=\operatorname{supp}(z)$. Using Property (F), we have that:

$$
\|h\| \leq \mathcal{F}\|h+w\|,
$$

for any $h$ and $w$ such that $h \cdot w=0$ and $\inf _{n \in \operatorname{supp}(w)}\left|e_{n}^{*}(w)\right| \geq\|\tilde{h}\|_{\infty}$.

Taking $D=\left\{n:\left|e_{n}^{*}(y)\right|=1\right\}$, observe that $y=P_{D^{c}}(y)+1_{\eta D}$, where $\eta \equiv\left\{\operatorname{sign}\left(e_{n}^{*}(y)\right)\right\}_{n}$. Then, if $A^{\prime} \subseteq A$,

$$
\left\|f+1_{A^{\prime}}\right\| \leq \mathcal{F}\left\|f+P_{D^{c}}(y)+1_{D}\right\| \leq \mathcal{F}\left(\|f+y\|+\left\|1_{\eta D}\right\|+\left\|1_{D}\right\|\right) .
$$

If we decompose $D^{c}=D_{1} \cup D_{2}$ such that:

$$
D_{1}=\left\{n:\left|e_{n}^{*}(y)\right|<1\right\}, \quad D_{2}=\left\{n:\left|e_{n}^{*}(y)\right|>1\right\},
$$

we obtain that:

$$
\left\|1_{\eta D}\right\| \leq\left\|f+P_{D_{1}}(y)+1_{\eta D}\right\|+\left\|f+P_{D_{1}}(y)\right\| \underset{(15)}{\leq} 2 \mathcal{F}\|f+y\| .
$$


Following the idea of (11), we can obtain that:

$$
\left\|1_{D}\right\| \leq 4 \mathcal{F}\left\|1_{\eta D}\right\| \underset{(17)}{\leq} 8 \mathcal{F}^{2}\|f+y\| .
$$

Using (17) and (18) in (16), we have:

$$
\left\|f+1_{A^{\prime}}\right\| \leq \mathcal{F}\left(1+2 \mathcal{F}+8 \mathcal{F}^{2}\right)\|f+y\| .
$$

Using Lemma 1, we obtain:

$$
\|f+z\| \leq \sup _{\varepsilon \in \mathcal{E}_{A}}\left\|f+1_{\mathcal{E} A}\right\| \underset{\text { Lemma } 2}{\leq} 5 \sup _{A^{\prime} \subseteq A}\left\|f+1_{A^{\prime}}\right\| \leq 5 \mathcal{F}\left(1+2 \mathcal{F}+8 \mathcal{F}^{2}\right)\|f+y\| .
$$

Therefore, the basis has Property $\left(\mathrm{F}^{*}\right)$ with $\mathcal{F}^{*} \leq 5 \mathcal{F}\left(1+2 \mathcal{F}+8 \mathcal{F}^{2}\right)$.

To finish this section, we give the following nice characterization of Property $\left(\mathrm{F}^{*}\right)$ that will be useful to show our main theorem.

Proposition 1. Let $\mathcal{B}$ be a basis in a Banach space $\mathbb{X}$. The following are equivalent:

(i) There is a positive constant $C$ such that:

$$
\left\|f+1_{\varepsilon A}\right\| \leq C\left\|f+g+1_{\eta B}\right\|,
$$

for any $f, g \in \mathbb{X}_{\text {fin }}$ such that $f \cdot g=0,\|\tilde{f}\|_{\infty} \leq 1$ and $\inf _{n \in \operatorname{supp}(g)}\left|e_{n}^{*}(g)\right| \geq\|\tilde{f}\|_{\infty}$, for any pair of finite sets $A$ and $B$ such that $A \cap B=\varnothing,|A| \leq|B|, \operatorname{supp}(f+g) \cap(A \cup B)=\varnothing$, and for any $\varepsilon \in \mathcal{E}_{A}, \eta \in \mathcal{E}_{B}$;

(ii) The basis has Property $\left(F^{*}\right)$ with constant $\mathcal{F}^{*}$;

(iii) There is a positive constant $C$ such that:

$$
\|f\| \leq C\left\|f-P_{A}(f)+y\right\|,
$$

for any $f, y \in \mathbb{X}_{\text {fin }}$ with $f \cdot y=0$ and $A \subseteq \operatorname{supp}(f)$ verifying:

(a) $\|\tilde{f}\|_{\infty} \leq 1$;

(b) $\inf _{n \in \operatorname{supp}(y)}\left|e_{n}^{*}(y)\right| \geq\|\tilde{f}\|_{\infty} ;$

(c) $|D| \geq|A|$, where $D=\left\{n \in \operatorname{supp}(y):\left|e_{n}^{*}(y)\right|=1\right\}$. we have:

Moreover, if we denote by $C_{1}$ and $C_{2}$ the least constants verifying (19) and (20), respectively,

$$
\mathcal{F}^{*} \leq C_{1}, C_{2} \leq \mathcal{F}^{*}, C_{1} \leq C_{2}
$$

Proof. First, we prove (i) $\Rightarrow$ (ii). Take $f, z, y \in \mathbb{X}_{f i n}$ as in the definition of Property $\left(\mathrm{F}^{*}\right)$ :

- $\quad f \cdot y=0, f \cdot z=0, z \cdot y=0$;

- $\max \left\{\|\tilde{f}\|_{\infty},\|\tilde{z}\|_{\infty}\right\} \leq 1$;

- $|D| \geq|\operatorname{supp}(z)|$, where $D=\left\{n:\left|e_{n}^{*}(y)\right|=1\right\}$;

- $\quad \inf _{n \in \operatorname{supp}(y)}\left|e_{n}^{*}(y)\right| \geq\|\tilde{f}\|_{\infty}$.

If $z=0$, just take $A=B=\varnothing$, and the proof is over. Consider now that $z \neq 0$, and take $\operatorname{supp}(z)=A$. If we divide $y=1_{\eta D}+P_{D^{c}}(y)$ with $\eta \equiv\left\{\operatorname{sign}\left(e_{n}^{*}(y)\right)\right\}$, we have for all $\varepsilon \in \mathcal{E}_{A}$,

$$
\left\|f+1_{\varepsilon A}\right\| \leq C_{1}\left\|f+P_{D^{c}(y)}+1_{\eta D}\right\|=C_{1}\|f+y\|
$$

Applying now Lemma 1, we obtain the result with $\mathcal{F}^{*} \leq C_{1}$. 
Now, we show that (ii) $\Rightarrow$ (iii). Of course, if $A=\varnothing$, the result is trivial. Take $f, y$, and $A$ as in (iii) with $A \neq \varnothing$ and $A \subseteq \operatorname{supp}(f)$. If in Property $\left(\mathrm{F}^{*}\right)$, we take $f^{\prime}=f-P_{A}(f)$, $z^{\prime}=1_{\varepsilon A}$ with $\varepsilon \in \mathcal{E}_{A}$ and $y^{\prime}=y$,

$$
\left\|f^{\prime}+z^{\prime}\right\|=\left\|f-P_{A}(f)+1_{\varepsilon A}\right\| \leq \mathcal{F}^{*}\left\|f^{\prime}+y^{\prime}\right\|=\mathcal{F}^{*}\left\|f-P_{A}(f)+y\right\|,
$$

so applying Item (ii) of Lemma 1, (iii) is proven with $C_{2} \leq \mathcal{F}^{*}$.

Finally, we give the proof to show that (iii) $\Rightarrow$ (i). Take $f, g \in \mathbb{X}_{\text {fin }}$ such that $f \cdot g=0$, $\|\tilde{f}\|_{\infty} \leq \inf _{n \in \operatorname{supp}(g)}\left|e_{n}^{*}(g)\right|,|A| \leq|B|<\infty, A \cap B=\varnothing, \operatorname{supp}(f+g) \cap(A \cup B)=\varnothing$, and $\varepsilon \in \mathcal{E}_{A}, \eta \in \mathcal{E}_{B}$.

Taking $f^{\prime}=f+1_{\varepsilon A}$ and $y=g+1_{\eta B}$,

$$
\left\|f+1_{\varepsilon A}\right\|=\left\|f^{\prime}\right\| \leq C_{2}\left\|f^{\prime}-P_{A}\left(f^{\prime}\right)+y\right\|=C_{2}\left\|f+g+1_{\eta B}\right\|,
$$

so the proof is over, and $C_{1} \leq C_{2}$.

\section{Proof of Theorem 5}

To prove Theorem 5, we used one of the most important tools in the world of quasigreedy bases: the truncation operator. To define this operator, we take $\alpha>0$ and define, first of all, the $\alpha$-truncation of $z \in \mathbb{C}$ :

$$
T_{\alpha}(z)=\alpha \operatorname{sign}(z), \text { if }|z| \geq \alpha,
$$

and:

$$
T_{\alpha}(z)=z, \text { if }|z| \leq \alpha .
$$

Now, it is possible to extend $T_{\alpha}$ to an operator in the space $\mathbb{X}$ by:

$$
T_{\alpha}(f)=\sum_{n \in \operatorname{supp}(f)} T_{\alpha}\left(e_{n}^{*}(f)\right) e_{n}=\sum_{n \in \Delta_{\alpha}} \alpha \frac{e_{n}^{*}(f)}{\left|e_{n}^{*}(f)\right|} e_{n}+\sum_{n \notin \Delta_{\alpha}} e_{n}^{*}(f) e_{n}
$$

where the set $\Delta_{\alpha}=\left\{n \in \mathbb{N}:\left|e_{n}^{*}(f)\right|>\alpha\right\}$. Of course, since $\Delta_{\alpha}$ is a finite set, $T_{\alpha}$ is well defined for all $f \in \mathbb{X}$.

Lemma 3 ([7] Lemma 2.5). Let $\mathcal{B}$ be a $C_{q}$-quasi-greedy basis in a Banach space. Then, the truncation operator is uniformly bounded, that is,

$$
\left\|T_{\alpha}(f)\right\| \leq C_{q}\|f\|, \forall \alpha>0, \forall f \in \mathbb{X} .
$$

Proof of Theorem 5. Assume that $\mathcal{B}$ is almost greedy with constant $C_{a l}$, and take $f, z$, and $y$ as in Property $\left(\mathrm{F}^{*}\right)$, then decompose $y=P_{B_{1}}(y)+P_{B_{2}}(y)+1_{\eta B}$, where $\eta \equiv\left\{\operatorname{sign}\left(e_{n}^{*}(y)\right)\right\}$, $B_{1} \cup B_{2}=B^{c}$ and:

$$
B_{1}=\left\{n:\left|e_{n}^{*}(y)\right|<1\right\}, \quad B_{2}=\left\{n:\left|e_{n}^{*}(y)\right|>1\right\} .
$$

Taking now $h:=f+1_{\varepsilon A}+P_{B_{2}}(y)+1_{\eta B}$ with $A=\operatorname{supp}(z), \varepsilon \in \mathcal{E}_{A}$, and $n=\left|B_{2}\right|+|B|$, we obtain:

$$
\begin{aligned}
\left\|f+1_{\varepsilon A}\right\| & =\left\|h-\mathcal{G}_{n}(h)\right\| \leq C_{a l}\left\|h-P_{A}(h)\right\|=C_{a l}\left\|f+P_{B_{2}}(y)+1_{\eta B}\right\| \\
& \leq C_{a l}\left(\|f+y\|+\left\|P_{B_{1}}(y)\right\|\right) \leq C_{a l}\left(\|f+y\|+\left\|f+P_{B_{1}}(y)\right\|+\|f\|\right) \\
& \leq C_{a l}\left(\|f+y\|+2 C_{a l}\|f+y\|\right) \\
& \leq C_{a l}\left(1+2 C_{a l}\right)\|f+y\| .
\end{aligned}
$$

Thus, applying Lemma 1 , the basis has Property $\left(\mathrm{F}^{*}\right)$ with constant $\mathcal{F}^{*} \leq C_{a l}\left(1+2 C_{a l}\right)$. 
Assume now that the basis has Property $\left(\mathrm{F}^{*}\right)$. Take $f \in \mathbb{X}_{f i n}, m \in \mathbb{N}, \mathcal{G}_{m}(f)=P_{G}(f)$, and $|A| \leq m$.

Consider now the elements $f^{\prime}=\frac{1}{t}\left(f-\mathcal{G}_{m}(f)\right)$ with $t=\min _{n \in G \backslash A}\left|e_{n}^{*}(f)\right|, B=$ $A \backslash G, y=1_{\eta(G \backslash A)}$, and $\eta \equiv\left\{\operatorname{sign}\left(e_{n}^{*}(f)\right)\right\}$. Of course, $f^{\prime} \cdot y=0,\left\|\tilde{f}^{\prime}\right\|_{\infty} \leq 1$ since $\left|e_{n}^{*}\left(f-\mathcal{G}_{m}(f)\right)\right| \leq t$ for $n \in G^{c}$ and $|G \backslash A| \geq|B|$. Then, applying these elements in Item (iii) of Proposition 1, we obtain the following:

$$
\begin{aligned}
\left\|f-\mathcal{G}_{m}(f)\right\| & =t\left\|f^{\prime}\right\| \leq t \mathcal{F}^{*}\left\|f^{\prime}-P_{B}\left(f^{\prime}\right)+y\right\| \\
& =\mathcal{F}^{*}\left\|f-P_{G}(f)-P_{A \backslash G}(f)+t 1_{\eta(G \backslash A)}\right\| \\
& =\mathcal{F}^{*}\left\|P_{(A \cup G)^{c}}\left(f-P_{A}(f)\right)+t 1_{\eta(G \backslash A)}\right\| .
\end{aligned}
$$

Since Property $\left(\mathrm{F}^{*}\right)$ implies that the basis is quasi-greedy with $C_{q} \leq \mathcal{F}^{*}$ (Theorems 7 and 8), applying Lemma 3,

$$
\left\|P_{(A \cup G)^{c}}\left(f-P_{A}(f)\right)+t 1_{\eta(G \backslash A)}\right\|=\left\|T_{t}\left(f-P_{A}(f)\right)\right\| \leq \mathcal{F}^{*}\left\|f-P_{A}(f)\right\| .
$$

Thus, by (23) and (22), the basis is almost greedy with constant $C_{a l} \leq\left(\mathcal{F}^{*}\right)^{2}$ for elements $f \in \mathbb{X}_{f i n}$. Now, applying Corollary 2, the results follow.

\section{Properties $\left(\mathrm{F}_{p}\right)$ and $\left(\mathrm{F}_{p}^{*}\right)$}

In all the results presented in Section 2, we can change democracy to conservativeness or super-conservativeness and Properties $(\mathrm{F})$ and $\left(\mathrm{F}^{*}\right)$ to Properties $\left(\mathrm{F}_{p}\right)$ and $\left(\mathrm{F}_{p}^{*}\right)$ and obtain the same results. Here, we only present the fundamental theorem that is the version of Theorem 7 to study how the constants change.

Theorem 9. $A$ basis $\mathcal{B}$ in a Banach space $\mathbb{X}$ has Property $\left(F_{p}\right)$ if and only if $\mathcal{B}$ is quasi-greedy and conservative. Moreover,

$$
\max \left\{\Delta_{c}, C_{q}\right\} \leq \mathcal{F}_{p} \leq 2+C_{q}+2 C_{q} \Delta_{c} .
$$

Proof. Assume that $\mathcal{B}$ has Property $\left(\mathrm{F}_{p}\right)$ with constant $\mathcal{F}_{p}$. Taking $A=\varnothing$, we have that:

$$
\|f\| \leq C\left\|f+1_{B}+g\right\|,
$$

for any $f, g$, and $B$ as in the definition of Property $\left(\mathrm{F}_{p}\right)$. Now, taking $B=\varnothing$ and considering $f^{\prime}:=f-\mathcal{G}_{m}(f)$ and $y=\mathcal{G}_{m}(f)$,

$$
\left\|f-\mathcal{G}_{m}(f)\right\|=\left\|f^{\prime}\right\| \leq \mathcal{F}_{p}\left\|f^{\prime}+g\right\|=\mathcal{F}_{p}\|f\|,
$$

so the basis is quasi-greedy for elements with finite support. Applying Corollary 1 , the basis is quasi-greedy with $C_{q} \leq \mathcal{F}_{p}$. Now, on the other hand, taking $f=g=0$, we obtain conservativeness with constant $\Delta_{c} \leq \mathcal{F}_{p}$.

Now, take $f, g, A$, and $B$ as in the definition of Property $\left(\mathrm{F}_{p}\right)$. If we have $g=g_{1}+g_{2}$ where:

$$
\begin{aligned}
& \operatorname{supp}\left(g_{1}\right)=\left\{n \in \operatorname{supp}(g):\left|e_{n}^{*}(g)\right|<1\right\} \\
\left\|f+1_{A}\right\| \leq & \left\|f+g+1_{B}\right\|+\left\|g+1_{B}\right\|+\left\|1_{A}\right\| \\
\leq & 2\left\|f+g+1_{B}\right\|+\|f\|+\left\|1_{A}\right\| \\
\leq & \left(2+C_{q}\right)\left\|f+g+1_{B}\right\|+\Delta_{c}\left\|1_{B}\right\| \\
\leq & \left(2+C_{q}\right)\left\|f+g+1_{B}\right\|+\Delta_{c}\left\|f+g_{1}+1_{B}\right\|+\Delta_{c}\left\|f+g_{1}\right\| \\
\leq & \left(2+C_{q}\right)\left\|f+g+1_{B}\right\|+2 C_{q} \Delta_{c}\left\|f+g+1_{B}\right\| \\
= & \left(2+C_{q}+2 C_{q} \Delta_{c}\right)\left\|f+g+1_{B}\right\| .
\end{aligned}
$$




\section{Proof of Theorem 6}

Proof of Theorem 6. Assume now that $\mathcal{B}$ is $C_{p}$-partially greedy, and prove Property $\left(\mathrm{F}_{p}^{*}\right)$. Take $f, z, y \in \mathbb{X}_{\text {fin }}$ satisfying from (i) to (iv) in the definition of Property $\left(\mathrm{F}_{p}^{*}\right)$. We write $y=1_{\eta D}+y_{1}+y_{2}$, where:

$$
\operatorname{supp}\left(y_{1}\right)=\left\{n \in \operatorname{supp}(y):\left|e_{n}^{*}(y)\right|<1\right\}, \operatorname{supp}\left(y_{2}\right)=\left\{n \in \operatorname{supp}(y):\left|e_{n}^{*}(y)\right|>1\right\},
$$

and $\eta \equiv\left\{\operatorname{sign}\left(e_{n}^{*}(y)\right)\right\}$. Consider $A=\operatorname{supp}(z)$.

If $A=\varnothing$, applying Theorem 4 , the basis is quasi-greedy with $C_{q} \leq C_{p}$, and we can conclude that $\|f\| \leq\|f+y\|$.

Assume now that $A \neq \varnothing$, and consider $m=\max A$, then define $B=\{1, \ldots, m\} \backslash A$. It is clear that $m=|A \cup B| \leq|B \cup D|$. Now, for any choice $\varepsilon \in \mathcal{E}_{A}$, define $h:=f+1_{\varepsilon}+y_{2}+$ $1_{\eta D}+1_{B}$.

Since partial greediness implies quasi-greediness with constant $C_{p}$ (see Theorem 4 ), we have:

$$
\begin{aligned}
\left\|f+1_{\varepsilon A}\right\| & =\left\|h-\mathcal{G}_{m}(h)\right\| \leq C_{p} \inf _{k \leq m}\left\|h-S_{k}(h)\right\| \leq C_{p}\left\|f+y_{2}+1_{\eta D}\right\| \\
& \leq C_{p}\left(\|f\|+\left\|y_{2}+1_{\eta D}\right\|\right) .
\end{aligned}
$$

For the first element of the sum, consider $w:=f+y$, and we have:

$$
\|f\|=\left\|w-\mathcal{G}_{n}(w)\right\|,
$$

with $n=|\operatorname{supp}(y)|$. Then, applying quasi-greediness, we obtain $\|f\| \leq C_{p}\|f+y\|$. For the second one, we write $w=f+y_{1}+y_{2}+1_{\eta D}$, and using quasi-greediness, we have:

$$
\left\|y_{2}+1_{\eta D}\right\|=\left\|\mathcal{G}_{m}(w)\right\| \leq\left(1+C_{p}\right)\|f+y\|,
$$

where $m=\left|\operatorname{supp}\left(y_{2}\right) \cup D\right|$.

Using both bounds, we obtain $\left\|f+1_{\varepsilon A}\right\| \leq C_{p}\left(1+2 C_{p}\right)\|f+y\|$. Because of Lemma 1, we conclude that $\|f+z\| \leq C_{p}\left(1+2 C_{p}\right)\|f+y\|$.

We now prove (b). Without loss of generality, we can assume that $f \in \mathbb{X}_{\text {fin }}$ using Corollary 3 and that $\|\tilde{f}\|_{\infty} \leq 1$. Start considering $A=\operatorname{supp}\left(\mathcal{G}_{m}(f)\right), k \leq m$, and $B=$ $\{1, \ldots, k\}$. If $A=B$, then the result is trivial. If $A \neq B$, we can decompose:

$$
f-\mathcal{G}_{m}(f)=P_{(A \cup B)^{c}}\left(f-S_{k}(f)\right)+P_{B \backslash A}(f) .
$$

Let $f^{\prime}=\frac{1}{t} P_{(A \cup B)^{c}}\left(f-S_{k}(f)\right)$ and $z=\frac{1}{t} P_{B \backslash A}(f)$ with $t=\min _{n \in A}\left|e_{n}^{*}(f)\right|$ and $y=$ $1_{\mathcal{\varepsilon}(A \backslash B)}$ with $\varepsilon \equiv\left\{\operatorname{sign}\left(e_{n}^{*}(f)\right\}\right.$. Of course, $f^{\prime} \cdot z=0, f^{\prime} \cdot y=0$, and $y \cdot z=0,\left\|\tilde{f}^{\prime}\right\|_{\infty} \leq 1$ since $\left|e_{n}^{*}\left(P_{(A \cup B)^{c}}\left(f-S_{k}(f)\right)\right)\right| \leq t$ for $n \in(A \cup B)^{c}$ and $|A \backslash B| \geq|B \backslash A|$. Then, $f^{\prime}, z$, and $y$ verify the items of Property $\left(\mathrm{F}_{p}^{*}\right)$, so:

$$
\left\|f-\mathcal{G}_{m}(f)\right\|=t\left\|f^{\prime}+z\right\| \leq t \mathcal{F}_{p}^{*}\left\|f^{\prime}+1_{\mathcal{\varepsilon}(A \backslash B)}\right\|=\mathcal{F}_{p}^{*}\left\|P_{(A \cup B)^{c}}\left(f-S_{k}(f)\right)+t 1_{\mathcal{\varepsilon}(A \backslash B)}\right\| .
$$

It turns out that:

$$
P_{(A \cup B)^{c}}\left(f-S_{k}(f)\right)+t 1_{\varepsilon A \backslash B}=T_{t}\left(f-S_{k}(f)\right),
$$

where $T_{t}$ is the $t$-truncation operator. Now, since Property $\left(\mathrm{F}_{p}^{*}\right)$ implies Property $\left(\mathrm{F}_{p}\right)$ with the same constant, because of Theorem 9 and Corollary 1 , the basis is quasi-greedy with constant $C_{q} \leq \mathcal{F}_{p}^{*}$. Then, applying Lemma 3, we have that $\left\|T_{t}\left(f-S_{k}(f)\right)\right\| \leq$ $\mathcal{F}_{p}^{*}\left\|f-S_{k}(f)\right\|$. All together, we obtain $\left\|f-\mathcal{G}_{m}(f)\right\| \leq\left(\mathcal{F}_{p}^{*}\right)^{2}\left\|f-S_{k}(f)\right\|$ for all $k \leq m$, and hence, $\mathcal{B}$ is partially greedy. 


\section{Annex}

In this Annex, we write the main lemmas about density that we used in the paper.

Lemma 4 ([12] Lemma 7.2). Let $\mathcal{B}$ be a basis for a Banach space $\mathbb{X}$. If $A$ is a greedy set for $f \in \mathbb{X}$, for every $\varepsilon>0$, there is $y \in \mathbb{X}_{\text {fin }}$ such that $\|f-y\| \leq \varepsilon$ and $A$ is a greedy set for $y$.

Corollary 1. Assume that $\mathcal{B}$ is a $C_{q}$-quasi-greedy basis of a Banach space $\mathbb{X}$ for elements with finite support. Then, $\mathcal{B}$ is quasi-greedy for every $f \in \mathbb{X}$.

Proof. Take $f \in \mathbb{X}$ and $A$ a greedy set of $f$ of order $m$. By Lemma 4, there is $y \in \mathbb{X}_{\text {fin }}$ such that $\|f-y\| \leq \varepsilon$ for every $\varepsilon>0$ with $A$ a greedy set for $y$. Then:

$$
\begin{aligned}
\left\|f-P_{A}(f)\right\| & =\left\|f-y-P_{A}(f)+y-P_{A}(y)+P_{A}(y)\right\| \\
& \leq\|f-y\|+\left\|y-P_{A}(y)\right\|+\left\|P_{A}(f-y)\right\| \\
& \leq \varepsilon\left(1+\left\|P_{A}\right\|\right)+C_{q}\|y\| \\
& \leq \varepsilon\left(1+\left\|P_{A}\right\|\right)+C_{q}\|f-y\|+C_{q}\|f\| \\
& \leq \varepsilon\left(1+C_{q}+\left\|P_{A}\right\|\right)+C_{q}\|f\| .
\end{aligned}
$$

Taking $\varepsilon \rightarrow 0$, we obtain the result.

Corollary 2. Let $\mathcal{B}$ be a basis for a Banach space $\mathbb{X}$. If $\mathcal{B}$ is an almost greedy basis for all $f \in \mathbb{X}_{\text {fin }}$ with constant $C_{a l}$, then the basis is almost greedy for every $f \in \mathbb{X}$ with the same constant.

Proof. Assume that $\mathcal{B}$ is almost greedy for elements with finite support. Take $f \in \mathbb{X}$ with $A$ a greedy set of order $m$. Applying Lemma 4, for any $\varepsilon>0$, there is $g \in \mathbb{X}_{f i n}$ such that $\|f-g\| \leq \varepsilon$ and $A$ a greedy set for $g$. Consider the set $B_{1}$ such that:

$$
\inf _{|B| \leq m}\left\|f-P_{B}(f)\right\|=\left\|f-P_{B_{1}}(f)\right\| .
$$

Case 1: $B_{1}=\varnothing$.

$$
\begin{aligned}
\left\|f-P_{A}(f)\right\| & =\left\|f-g+g-P_{A}(f)-P_{A}(g)+P_{A}(g)\right\| \\
& \leq\|f-g\|+\left\|g-P_{A}(g)\right\|+\left\|P_{A}(f-g)\right\| \\
& \leq \varepsilon\left(1+\left\|P_{A}\right\|\right)+C_{a l} \inf _{|B| \leq m}\left\|g-P_{B}(g)\right\| \\
& \leq \varepsilon\left(1+\left\|P_{A}\right\|\right)+C_{a l}\|g\| \\
& \leq \varepsilon\left(1+\left\|P_{A}\right\|\right)+C_{a l}\|f-g\|+C_{a l}\|f\| \\
& \leq \varepsilon\left(1+C_{a l}+\left\|P_{A}\right\|\right)+C_{a l}\|f\| .
\end{aligned}
$$

Taking $\varepsilon \rightarrow 0$, we obtain the result.

Case 2: $B_{1} \neq \varnothing$.

$$
\begin{aligned}
\left\|f-P_{A}(f)\right\| & =\left\|f-g+g-P_{A}(f)-P_{A}(g)+P_{A}(g)\right\| \\
& \leq\|f-g\|+\left\|g-P_{A}(g)\right\|+\left\|P_{A}(f-g)\right\| \\
& \leq \varepsilon\left(1+\left\|P_{A}\right\|\right)+C_{a l} \inf _{|B| \leq m}\left\|g-P_{B}(g)\right\| \\
& \leq \varepsilon\left(1+\left\|P_{A}\right\|\right)+C_{a l}\left\|g-P_{B_{1}}(g)\right\| \\
& \leq \varepsilon\left(1+\left\|P_{A}\right\|\right)+C_{a l}\left\|g-f+f-P_{B_{1}}(g)+P_{B_{1}}(f)-P_{B_{1}}(f)\right\| \\
& \leq \varepsilon\left(1+\left\|P_{A}\right\|+C_{a l}\right)+C_{a l}\left\|P_{B_{1}}(f-g)\right\|+\left\|f-P_{B_{1}}(f)\right\| \\
& \leq \varepsilon\left(1+\left\|P_{A}\right\|+C_{a l}+C_{a l}\left\|P_{B_{1}}\right\|\right)+\left\|f-P_{B_{1}}(f)\right\|
\end{aligned}
$$

Taking $\varepsilon \rightarrow 0$, we obtain the result. 
With the same arguments, it is straightforward to show the next result.

Corollary 3. Let $\mathcal{B}$ be a basis for a Banach space $\mathbb{X}$. If $\mathcal{B}$ is a partially greedy basis for all $f \in \mathbb{X}_{\text {fin }}$ with constant $C_{p}$, then the basis is partially greedy for every $f \in \mathbb{X}$ with the same constant.

Lemma 5 ([10] Lemma 3.2). Let $\mathbb{X}$ be a Banach space. Suppose $D$ is a finite subset of $\mathbb{N}$, and $f \in \mathbb{X} \backslash\{0\}$ satisfies $\operatorname{supp}(f) \cap D=\varnothing$. Then, for any $\varepsilon>0$ there is $y \in \mathbb{X}_{\text {fin }}$, so that $\|f-y\|<\varepsilon, \operatorname{supp}(y) \cap D=\varnothing$, and $\|\tilde{f}\|_{\infty}=\|\tilde{y}\|_{\infty}$.

Author Contributions: All authors have contributed equally to this work. All authors have read and agreed to the published version of the manuscript.

Funding: The first author was supported by Grants PID2019-105599GB-I00 (Agencia Estatal de Investigación, Spain) and 20906/PI/18 from Fundación Séneca (Región de Murcia, Spain). The second author was supported by the research project PGC2018-098321-B-I00 (Ministerio de Ciencia, Innovación y Universidades, Spain).

Institutional Review Board Statement: Not applicable.

Informed Consent Statement: Not applicable.

Data Availability Statement: Not applicable.

Conflicts of Interest: The authors declare no conflict of interest.

\section{References}

1. Konyagin, S.V.; Temlyakov, V.N. A remark on greedy approximation in Banach spaces. East J. Approx. 1999, 5, 365-379

2. Wojtaszczyk, P. Greedy algorithm for general biorthogonal systems. J. Approx. Theory 2000, 107, 293-314 [CrossRef]

3. Albiac, F.; Ansorena, J.L.; Berná, P.M.; Wojtaszczyk, P. Greedy approximation for biorthogonal systems in quasi-Banach spaces. Dissertatione Math. 2021, 560, 1-88

4. Dilworth, S.J.; Kalton, N.J.; Kutzarova, D.; Temlyakov, V.N. The Thresholding Greedy Algorithm, Greedy Bases, and Duality. Constr. Approx. 2003, 19, 575-597. [CrossRef]

5. Berasategui, M.; Berná, P.M.; Lassalle, S. Strong Partially Greedy Bases and Lebesgue-Type Inequalities. Constr. Approx. 2021. [CrossRef]

6. Dilworth, S.J.; Kutzarova, D.; Odell, E.; Schlumprecht, T.; Zsak, A. Renorming spaces with greedy bases. J. Approx. Theory 2014, 188, 39-56. [CrossRef]

7. Berná, P.M.; Blasco, O.; Garrigós, G. Lebesgue inequalities for greedy algorithm in general bases. Rev. Mat. Complut. 2017, 30, 369-392. [CrossRef]

8. Berná, P.M.; Blasco, Ó. Characterization of greedy bases in Banach spaces. J. Approx. Theory 2017, 217, 28-39 [CrossRef]

9. Garrigós, G.; Hernández, E.; Oikhberg, T. Lebesgue-type inequalities for quasi-greedy bases. Constr. Approx. 2013, 38, 447-470. [CrossRef]

10. Berná, P.M.; Dilworth, S.J.; Kutzarova, D.; Oikhberg, T.; Wallis, B. The weighted property (A) and the greedy algorithm. J. Approx. Theory 2019, 248, 105300. [CrossRef]

11. Berná, P.M. Thresholding Greedy Algorithms in Banach Spaces. Ph.D. Thesis . Universidad Autónoma de Madrid, Spain, 2019. Available online: https:/ / repositorio.uam.es/handle/10486/688914 (accessed on 29 July 2019).

12. Berasategui, M.; Berná, P.M. Quasi-greedy bases for sequence with gaps. Nonlinear Anal. 2021, 208, 112294. [CrossRef] 